\title{
Relationship between Red Cell Distribution Width and Short-term Outcomes in Acute Coronary Syndrome in a Chinese Population
}

\author{
Yan-Ling Wang ${ }^{1}$, Qi Hua ${ }^{1}$, Cheng-Rui Bai $^{2}$ and Qing Tang ${ }^{1}$
}

\begin{abstract}
Objective This study was undertaken to determine the relationship between red cell distribution width (RDW) and short-term outcomes in acute coronary syndrome (ACS) in a Chinese population.

Methods and Patients A total of 1,654 patients with ACS on admission were divided into four groups according to quartiles of baseline RDW. The relationships between RDW and one-month cardiac mortality as well as heart failure and recurrent infarction were assessed.

Results Higher RDW values were associated with increased one-month cardiac mortality (quartile 1: $0.2 \%$; quartile 2: $0.6 \%$; quartile 3: $3.3 \%$; quartile $4: 8.1 \%$; $<0.001$ ) and one-month heart failure and recurrent infarction (quartile 1: 2.1\%; quartile 2: $2.7 \%$; quartile 3: $3.6 \%$; quartile 4: $15.2 \%$; $\mathrm{p}<0.001$ ). Logistic regression analysis revealed that RDW independently predicted for cardiac mortality (OR: 2.116, 95\% CI: 1.427-3.137, $\mathrm{p}<0.001)$ and heart failure and recurrent infarction (OR: $2.134,95 \%$ CI: $1.602-2.844, \mathrm{p}<0.001)$ during a onemonth follow-up in patients with ACS.

Conclusion The present study indicates that elevated RDW could predict an increased risk of short-term adverse outcomes in patients with ACS.
\end{abstract}

Key words: red cell distribution width, acute coronary syndrome, cardiac mortality, heart failure

(Intern Med 50: 2941-2945, 2011)

(DOI: 10.2169/internalmedicine.50.6407)

\section{Introduction}

Red cell distribution width (RDW), a measure of red blood cell size heterogeneity, is reported as part of the routine blood cell count. Recent studies have reported that higher RDW is associated with long-term mortality in patients with heart failure, stable coronary disease, and also in the general population (1-3). However, there is no information regarding the short-term prognostic significance of RDW in Chinese patients with acute coronary syndrome (ACS). In this study, we investigated the association between RDW and the risk of short-term mortality and adverse cardiovascular outcomes in patients with ACS in a Chinese population.

\section{Materials and Methods}

\section{Patients}

From January 2006 to May 2010, 1,654 consecutive patients (961 men and 693 women) with ACS who were admitted to our institute were enrolled; these included STsegment elevation myocardial infarction [(STEMI) 374 patients], non-ST-segment elevation myocardial infarction (NSTEMI) 323 patients], and unstable angina pectoris [(UA) 957 patients]. Diagnostic criteria of STEMI is 1) typical, prolonged chest pain at rest (>30 minutes); 2) ST-segment elevation $\geq 0.2 \mathrm{mV}$ at the $\mathrm{J}$ point in 2 or more contiguous, precordial leads, or $\geq 0.2 \mathrm{mV}$ in 2 or more adjacent limb leads on the standard 12-lead electrocardiogram (ECG); and

${ }^{1}$ Department of Cardiology, Xuanwu Hospital, Capital Medical University, China, and ${ }^{2}$ Department of Orthopedics, Beijing Friendship Hospital, Capital Medical University, China

Received for publication August 18, 2011; Accepted for publication September 7, 2011

Correspondence to Dr. Qi Hua, huaqi5371@ medmail.com.cn 
3) increased serial serum markers of myocardial damage [>2-fold increase over the upper normal range required for creatine kinase (CK) and troponin-I (TnI)] (4). UA/NSTEMI is defined by electrocardiographic (ECG) ST-segment depression or prominent $\mathrm{T}$-wave inversion and/or positive biomarkers of necrosis (e.g., troponin) in the absence of STsegment elevation and in an appropriate clinical setting (chest discomfort or anginal equivalent) (5). Patients with equivocal or uninterpretable ECGs (i.e., left bundle branch block, paced rhythm, or persistent ST-segment elevation after a previous MI) were not included in the study. Basic drug treatment for ACS included antiplatelet agents, Betablockers, angiotensin-converting enzyme inhibitor (ACE-I)/ angiotensin receptor blocker (ARB) and statins. Qualifying patients received thrombolytic therapy (tissue-type plasminogen activator or streptokinase) or underwent percutaneous coronary intervention (PCI) according to the discretion of the attending cardiologist. Each patient underwent PCI using standard techniques performed by experienced interventional cardiologists. Bleeding of PCI was small.

Furthermore, this study did not include patients with a history of anemia and blood transfusions, chronic heart failure, neoplastic, renal, liver, or thyroid disease, or patients receiving treatment with anti-inflammatory drugs. Patients with acute or chronic infections and autoimmune disease were also excluded from the study. The study protocol was approved by the ethics committee of our institution, and written informed consent was obtained from all participating subjects.

\section{Clinical data collection}

A special questionnaire was used to collect information on lifestyle, environmental factors, and medical history of the study population. Patients who reported smoking at least 1 cigarette per day for at least 1 year were defined as current smokers. Diabetes mellitus was defined as a previous diagnosis, use of diet or antidiabetic medicines, or fasting venous blood glucose level $\geq 126 \mathrm{mg} / \mathrm{dL}$ on 2 occasions in previously untreated patients. Patients who received medications for hypertension, or those with seated systolic blood pressure $\geq 140 \mathrm{mmHg}$ and/or diastolic blood pressure $\geq 90$ $\mathrm{mmHg}$ on at least 3 separate clinic visits were also identified. Height, weight, and body mass index (BMI) was calculated as the weight in kilograms divided by the square of the height in meters. Left ventricular ejection fractions (LVEF) were measured with Doppler echocardiography during the 3 days after admission.

\section{Blood collection and assays}

On admission, venous blood was obtained from all patients enrolled in the study. RDW, brain natriuretic peptide (BNP), high-sensitivity C-reactive protein (hs-CRP) and other biochemistry measurements were carried out by our biochemistry department using standard methods. Within 24 hours after admission, creatine phosphokinase (CPK) was measured for 6 hours and the maximum was recorded. The normal reference range for RDW in this laboratory is $0.0 \%$ to $15.0 \%$.

\section{Study endpoints}

All patients were followed-up for up to 1 month after discharge using a standardized protocol that included outpatient visits, telephone contacts, and the recording of recurrent cardiac events. The primary end point was cardiac death and the second outcomes were readmission for management of heart failure and recurrent infarction. A death was classified as cardiac if the predominant and immediate cause was related to myocardial infarction (MI) or ischemia, arrhythmia, refractory congestive heart failure, or sudden death. Heart failure was defined by the presence of new symptoms of dyspnea with pulmonary venous congestion on x-ray with interstitial or alveolar edema requiring hospitalization.

\section{Statistical analysis}

The study population was divided into 4 groups according to quartiles of baseline RDW. The lowest quartile served as the reference group. Data are expressed as means \pm standard deviation (SD) for normally distributed variables. Nonnormally distributed values are expressed as median and interquartile ranges. Qualitative data are presented as numbers (percentages).The comparison of the data among the four groups was performed by a one-way ANOVA or KruskalWallis test for continuous variables and by a chi-square test for categorical variables. Correlations between RDW and other biochemistry measurements were assessed using Spearman's rank correlations test for these continuous variables with non-normal distribution, and logistic regression was used to analyze the relationship between RDW and short-term adverse outcomes of ACS. Logistic regression was adjusted for RDW categorized according to quartiles of distribution, BNP $>100 \mathrm{ng} / \mathrm{mL}, \mathrm{LVEF}<40 \%$, age $>65$ years, gender, history of hypertension and diabetes, smoking status, BMI, AMI on presentation, serum creatinine, peak CPK, number of diseased vessels $\geq 2$, Troponin-I (TNI), hsCRP, white blood cell (WBC), hemoglobin and medical therapy (antiplatelet agents, beta-blockers, ACE-inhibitor/ $\mathrm{ARB}$, statins, thiazide diuretic and reperfusion therapy). A value of $\mathrm{p}<0.05$ was considered statistically significant. All calculations were performed using SPSS statistical software for windows V11.5 (SPSS, Inc., Chicago, IL, USA).

\section{Results}

\section{Clinical characteristics}

Compared with the control subjects, patients with higher RDW values were significantly older $(\mathrm{p}<0.001)$ and were observed with a significantly higher incidence in men than women $(\mathrm{p}<0.001)$. They were more likely to have history of hypertension $(\mathrm{p}<0.001)$ and diabetes mellitus $(\mathrm{p}=0.007)$ and to be current smoker $(\mathrm{p}<0.001)$. Meanwhile, there were significant higher levels of BMI $(p=0.005)$, creatinine $(p<$ 
Table 1. Baseline Characteristics of the Study Population, Mean \pm SD, Number (\%), or Median (Interquartile Range)

\begin{tabular}{|c|c|c|c|c|c|}
\hline & & & W quartiles & & \\
\hline Characteristic & $\begin{array}{l}\leq 12.1 \\
(n=419)\end{array}$ & $\begin{array}{l}12.2-12.8 \\
(n=364)\end{array}$ & $\begin{array}{l}12.9-13.2 \\
(\mathrm{n}=463)\end{array}$ & $\begin{array}{l}\geq 13.3 \\
(n=408)\end{array}$ & $\mathrm{p}$ value \\
\hline Age (y) & $62.62 \pm 11.90$ & $63.02 \pm 11.90$ & $66.30 \pm 10.78$ & $67.06 \pm 12.92$ & $<0.001$ \\
\hline Gender (male) & $257(61.3 \%)$ & $118(32.4 \%)$ & $321(69.3 \%)$ & $265(65.0 \%)$ & $<0.001$ \\
\hline $\begin{array}{l}\text { Hypertension } \\
\text { (history) }\end{array}$ & $241(72.8 \%)$ & $242(79.6 \%)$ & $348(85.1 \%)$ & $307(80.4 \%)$ & $<0.001$ \\
\hline Diabetes mellitus & $83(19.7 \%)$ & $82(22.4 \%)$ & $128(27.7 \%)$ & $113(27.7 \%)$ & 0.007 \\
\hline Current smoker & $137(32.7 \%)$ & $114(31.3 \%)$ & $207(44.7 \%)$ & $195(47.8 \%)$ & $<0.001$ \\
\hline $\operatorname{BMI}\left(\mathrm{kg} / \mathrm{m}^{2}\right)$ & $24.68 \pm 3.43$ & $24.64 \pm 3.32$ & $25.37 \pm 3.09$ & $25.50 \pm 3.54$ & 0.005 \\
\hline AMI on presentation & $171(41.2 \%)$ & $153(42.3 \%)$ & $194(42.6 \%)$ & $179(44.3 \%)$ & 0.810 \\
\hline $\operatorname{Scr}(\mu \mathrm{mol} / \mathrm{L})$ & $69.83 \pm 24.66$ & $70.44 \pm 24.74$ & $76.48 \pm 29.21$ & $84.94 \pm 41.17$ & $<0.001$ \\
\hline Peak CPK (IU/L) & $97.0(59.0-657.0)$ & $108.0(60.0-1037.0)$ & $117.0(61.0-949.0)$ & $113.0(54.0-1230.0)$ & 0.429 \\
\hline TNI (ng/mL) & $0.21(0.01-2.36)$ & $0.12(0.05-2.27)$ & $2.84(0.05-9.19)$ & $2.70(0.05-17.9)$ & $<0.001$ \\
\hline hs-CRP (mg/L) & $1.38(0.03-4.02)$ & $1.52(0.12-6.50)$ & $1.68(0.01-5.29)$ & $2.31(0.18-12.80)$ & $<0.001$ \\
\hline BNP (ng/mL) & $79.7(0.98-294)$ & $54.7(5.20-175)$ & $51.6(0.1-123)$ & $280.0(0.17-774)$ & $<0.001$ \\
\hline $\mathrm{WBC}\left(\times 10^{9} / \mathrm{L}\right)$ & $7.86 \pm 3.75$ & $7.95 \pm 3.19$ & $7.89 \pm 2.78$ & $8.10 \pm 3.20$ & 0.718 \\
\hline $\operatorname{HGB}(\mathrm{g} / \mathrm{L})$ & $139.11 \pm 49.93$ & $133.57 \pm 13.72$ & $133.11 \pm 38.49$ & $127.96 \pm 20.77$ & 0.016 \\
\hline LVEF (\%) & $62.60 \pm 11.67$ & $61.74 \pm 9.71$ & $60.92 \pm 9.08$ & $58.19 \pm 12.30$ & $<0.001$ \\
\hline $\begin{array}{l}\text { Number of diseased } \\
\text { vessels } \geq 2 \text { (stenosis } \\
\geq 50 \%)\end{array}$ & $136(32.5 \%)$ & $115(31.6 \%)$ & $157(33.9 \%)$ & $142(34.8 \%)$ & 0.776 \\
\hline Medication & - & - & - & - & - \\
\hline Antiplatelet agents & $356(85.6 \%)$ & $306(84.3 \%)$ & $389(84.1 \%)$ & $318(78.6 \%)$ & $<0.001$ \\
\hline Beta-blockers & $360(86.3 \%)$ & $302(83.1 \%)$ & $370(80.5 \%)$ & $330(81.2 \%)$ & $<0.001$ \\
\hline ACE-inhibitor/ARB & $348(83.4 \%)$ & $288(79.6 \%)$ & $384(83.2 \%)$ & $339(83.1 \%)$ & 0.360 \\
\hline Statins & $369(88.2 \%)$ & $324(89.3 \%)$ & $403(87.7 \%)$ & $359(88.9 \%)$ & $<0.001$ \\
\hline Thiazide diuretic & $8(2.3 \%)$ & $15(3.5 \%)$ & $14(3.4 \%)$ & $16(4.3 \%)$ & 0.194 \\
\hline Reperfusion therapy & $235(56.1 \%)$ & $200(55.7 \%)$ & $259(56.2 \%)$ & $220(54.4 \%)$ & 0.926 \\
\hline
\end{tabular}

Table 2. Spearman's Correlations Analysis between RDW and Other Biochemistry Markers ( $n=1654)$

\begin{tabular}{ccc}
\hline Variable & \multicolumn{2}{c}{ RDW } \\
\cline { 2 - 3 } & r value & p value \\
\hline BNP & 0.211 & $<0.001$ \\
hs-CRP & 0.070 & 0.018 \\
TNI & 0.110 & 0.003 \\
Scr & 0.118 & $<0.001$ \\
WBC & -0.003 & 0.913 \\
HGB & -0.111 & 0.001 \\
\hline Abbreviations: BNP, brain natriuretic peptide; hs-CRP, high-sensitivity C-reactive \\
protein; TnI, Troponin-I; Scr, serum creatinine; WBC, white blood cell; HGB, \\
hemoglobin.
\end{tabular}

$0.001)$, troponin-I $(\mathrm{p}<0.001)$, hs-CRP $(\mathrm{p}<0.001)$ and BNP (p $<0.001)$ in patients with higher RDW values. They had a lower level of hemoglobin $(\mathrm{p}=0.016)$ and LVEF $(\mathrm{p}<0.001)$. However, there was no significant difference in AMI on presentation, peak CPK, WBC, medication of ACE-I/ARB and thiazide diuretic, number of diseased vessels $\geq 2$ or reperfusion therapy among the four groups. Clinical characteristics of patients according to quartiles of baseline RDW are presented in Table 1.

\section{Correlations between RDW and other biochemistry markers}

In the whole study population, using RDW as a continuous variable, there was a slight correlation between RDW and BNP $(\mathrm{r}=0.211, \mathrm{p}<0.001)$. The correlation coefficient between RDW and hs-CRP was $0.070(\mathrm{p}=0.018)$. Correlations between RDW and other biochemistry markers are presented in Table 2.

\section{Relationship between RDW and cardiac mortality at 1 month}

Follow-up information was available for 1,604 patients (97.0\%) from the 1,654 ACS patients at 1 month. There were a total of 50 deaths $(3.1 \%)$ classified as cardiac in etiology. Cardiac death was more likely to occur in patients with higher RDW values (quartile 1: $0.2 \%$; quartile 2: $0.6 \%$; quartile $3: 3.3 \%$; quartile $4: 8.1 \%$; $<<0.001$ ). After multivariable adjustment for other important covariates mentioned above, the variables remaining in the equation were RDW (quartiles), BNP $>100 \mathrm{ng} / \mathrm{mL}$ and $\mathrm{LVEF}<40 \%$ (Table 3). Using baseline RDW as a categorical variable according to quartiles, the adjusted Odds Ratio for cardiac mortality in- 
Table 3. Logistic Regression Analysis for Predictors of Cardiac Mortality

\begin{tabular}{lccc}
\hline Variable & Odds Ratio & $95 \%$ Confidence Interval & $\mathrm{p}$ value \\
\hline BNP $>100$ & 3.510 & $1.221-10.093$ & 0.020 \\
RDW(quartiles) & 2.116 & $1.427-3.137$ & $<0.001$ \\
LVEF $<40 \%$ & 4.149 & $2.001-8.602$ & $<0.001$ \\
\hline Abbreviations: BNP, brain natriuretic peptide ; RDW, Red cell distribution width; \\
LVEF, left ventricular ejection fraction.
\end{tabular}

crease was 2.116 (95\% CI: 1.427-3.137, p<0.001). Meanwhile, BNP $>100 \mathrm{ng} / \mathrm{mL}$ (OR: 3.510, 95\% CI: 1.221-10.093, $\mathrm{p}=0.020$ ) and $\mathrm{LVEF}<40 \%$ (OR: 4.149, 95\% CI: 2.001-8.602, $\mathrm{p}<0.001$ ) were identified as significant independent predictors of cardiac mortality by logistic regression analysis.

\section{Relationship between RDW and heart failure and re- current infarction at 1 month}

During one-month follow-up, 95 patients $(5.9 \%)$ were readmitted for management of heart failure and recurrent infarction. Higher RDW values were associated with increased prevalence of heart failure and recurrent infarction: (quartile 1: $2.1 \%$; quartile 2 : $2.7 \%$; quartile $3: 3.6 \%$; quartile 4 : $15.2 \% ; \mathrm{p}<0.001)$. Based on multivariable logistic regression analysis, RDW (quartiles) was found to be an independent predictor of heart failure and recurrent infarction at 1 month (OR: 2.134, 95\% CI: $1.602-2.844, \mathrm{p}<0.001)$. In addition, logistic regression analysis also identified age $>65$ years (OR: 2.010, 95\% CI: $1.135-3.560, \mathrm{p}=0.017)$ and diabetes mellitus (OR: $2.279,95 \%$ CI: 1.345-3.862, $\mathrm{p}=0.002$ ) and $\mathrm{LVEF}<40 \%$ (OR: $5.009,95 \%$ CI: $2.694-9.316, p<0.001$ ), as significant independent predictors of heart failure and recurrent infarction at 1 month (Table 4).

\section{Discussion}

The present study showed that adverse events were more likely to occur in patients with ACS during short-term follow up if they had higher RDW values. Furthermore, RDW was positively correlated with BNP and hs-CRP. Logistic regression analysis revealed that RDW was an independent predictor for cardiac mortality and the onset of heart failure and recurrent infarction during a one-month follow-up in patients with ACS. The results suggest that measuring the RDW level may provide valuable information for short-term risk stratification of ACS. These findings are also notable given that RDW is widely available to clinicians as part of the complete blood count and therefore incurs no additional costs, in contrast to other novel markers of cardiovascular risk.

RDW reflects the variability in circulating red blood cell size. It is based on the width of red blood cell volume distribution curve, with larger values indicating greater variability $(6,7)$, RDW is typically increased in conditions of ineffective red cell production (such as iron deficiency, B12 or folate deficiency, and hemoglobinopathies), increased red cell destruction (such as hemolysis), and with blood transfusion $(8,9)$. However, all of these conditions were not pre-
Table 4. Logistic Regression Analysis for Predictors of Heart Failure and Recurrent Infarction

\begin{tabular}{lccc}
\hline Variable & Odds Ratio & $95 \%$ Confidence Interval & $\mathrm{p}$ value \\
\hline Age $>65$ years & 2.010 & $1.135-3.560$ & 0.017 \\
Diabetes mellitus & 2.279 & $1.345-3.862$ & 0.002 \\
RDW (quartiles) & 2.134 & $1.602-2.844$ & $<0.001$ \\
LVEF $<40 \%$ & 5.009 & $2.694-9.316$ & $<0.001$ \\
\hline Abbreviations: RDW, Red cell distribution width; LVEF, left ventricular ejection \\
fraction.
\end{tabular}

sent in our study population and adjustment for multiple potential confounders attenuated but did not eliminate the strong association between higher RDW levels and the adverse clinical outcomes.

The mechanism by which elevated values of RDW are associated with adverse outcomes is unknown. It has been suggested that RDW may reflect other known markers of prognosis in heart failure, such as inflammatory cytokines, which may affect bone marrow function and iron metabolism $(10,11)$. Inflammatory cytokines have been found to suppress the maturation of erythrocytes, allowing juvenile erythrocytes to enter into circulation and thereby leading to an increase in heterogeneity of the size $(12,13)$. Furthermore, higher RDW may reflect enhanced erythropoiesis resulting from elevated circulating levels of neurohumoral mediators $(14,15)$. There is increasing evidence that a chronic inflammatory state and neurohumoral activation each contribute to adverse clinical outcomes in patients with $\operatorname{ACS}(16,17)$.

In the present study, RDW was positively correlated with BNP $(r=0.211, p<0.001)$ and hs-CRP $(r=0.070, p=0.018)$. Although BNP is produced mainly by the ventricle, a variety of vasoconstrictive neurohormones including angiotensin II and norepinephrine have been found to directly enhance BNP secretion $(18,19)$. These aspects may explain RDW was positively correlated with BNP in our study. However, we can see that the correlation coefficients between RDW and BNP, hs-CRP were both low; the time of blood collection might affect the values. Thus, in this study we could not reveal the exact mechanism of the association of higher RDW and increased adverse outcomes in patients with ACS. Additional studies should be performed to determine the underlying factors that affect RDW.

The present study had several limitations. First, this was a post hoc observational analysis, and therefore we cannot rule out the possibility of residual confounding. However, the hypothesis that RDW levels would be associated with adverse outcomes was formulated before analyses were started, reducing the risk of spurious conclusions. Second, although we speculate that neurohumoral activation may be a mechanistic link between increased RDW and adverse outcomes in this population, we did not measure the plasma level of angiotensin II or norepinephrine in our patients. Third, we did not have data on the changes in RDW during the hospital course. Changes in RDW during the hospital course for ACS may predict short-term clinical outcomes better (20). 
In conclusion, in patients with ACS, elevated RDW could predict an increased risk of short-term adverse outcomes. More studies are needed to address the mechanism and treatment.

The authors state that they have no Conflict of Interest (COI).

\section{Acknowledgement}

The authors would like to express their sincere thanks to the staff at the Department of Cardiology, Xuanwu Hospital, Capital Medical University, Bejing, China, for their kind cooperation when conducting this study. This study was supported by Capital Medical Development Fund Project (2009-1054), Beijing Municipal Science and Technology Commission Funded Project (Z 101107050210016) and 973Program (2012CB517805).

\section{References}

1. Felker GM, Allen LA, Pocock SJ, et al. Red cell distribution width as a novel prognostic marker in heart failure: data from the CHARM Program and the Duke Databank. J Am Coll Cardiol 50: 40-47, 2007.

2. Tonelli M, Sacks F, Arnold M, et al. Relation between red blood cell distribution width and cardiovascular event rate in people with coronary disease. Circulation 117: 163-168, 2008.

3. Lippi G, Targher G, Montagnana M, Salvagno GL, Zoppini G, Guidi GC. Relation between red blood cell distribution width and inflammatory biomarkers in a large cohort of unselected outpatients. Arch Pathol Lab Med 133: 628-632, 2009.

4. Antman EM, Anbe DT, Armstrong PW, et al. ACC/AHA guidelines for the management of patients with st-elevation myocardial infarction-Executive summary. Circulation 110: 588-636, 2004.

5. Sami S, Willerson JT. Contemporary treatment of unstable angina and non-ST-segment-elevation myocardial infarction (part 2). Tex Heart Inst J 37: 262-275, 2010.

6. Uyarel H, Ergelen M, Cicek G, et al. Red cell distribution width as a novel prognostic marker in patients undergoing primary angioplasty for acute myocardial infarction. Coron Artery Dis 22: 138-144, 2011.

7. Zalawadiya SK, Veeranna V, Niraj A, Pradhan J, Afonso L. Red cell distribution width and risk of coronary heart disease events. Am J Cardiol 106: 988-993, 2010.

8. Förhécz Z, Gombos T, Borgulya G, Pozsonyi Z, Prohászka Z, Jánoskuti L. Red cell distribution width in heart failure: prediction of clinical events and relationship with markers of ineffective erythropoiesis, inflammation, renal function, and nutritional state.. Am Heart J 158: 659-666, 2009.

9. Chen PC, Sung FC, Chien KL, Hsu HC, Su TC, Lee YT. Red blood cell distribution width and risk of cardiovascular events and mortality in a community cohort in taiwan. Am J Epidemiol 171: 214-220, 2010.

10. Pfister R, Diedrichs H, Schiedermair A, et al. Prognostic impact of NT-proBNP and renal function in comparison to contemporary multi-marker risk scores in heart failure patients. Eur J Heart Fail 10: 315-320, 2008.

11. Dickstein K, Cohen-Solal A, Filippatos G, et al. ESC guidelines for the diagnosis and treatment of acute and chronic heart failure 2008: the Task Force for the Diagnosis and Treatment of Acute and Chronic Heart Failure 2008 of the European Society of Cardiology. Eur J Heart Fail 10: 933-989, 2008.

12. Pascual-Figal DA, Bonaque JC, Redondo B, et al. Red blood cell distribution width predicts long-term outcome regardless of anaemia status in acute heart failure patients. Eur J Heart Fail 11: 840846, 2009.

13. Al-Najjar Y, Goode KM, Zhang J, Cleland JG, Clark AL. Red cell distribution width: an inexpensive and powerful prognostic marker in heart failure. Eur J Heart Fail 11: 1155-1162, 2009.

14. Allen LA, Felker GM, Mehra MR, et al. Validation and potential mechanisms of red cell distribution width as a prognostic marker in heart failure. J Cardiac Fail 16: 230-238, 2010.

15. Masson S, Latini R, Anand IS, et al. Prognostic value of changes in N-terminal pro-brain natriuretic peptide in Val-HeFT (Valsartan Heart Failure Trial). J Am Coll Cardiol 52: 997-1003, 2008.

16. Nabais S, Losa N, Gaspar A, et al. Association between red blood cell distribution width and outcomes at six months in patients with acute coronary syndromes. Rev Port Cardiol 28: 905-924, 2009.

17. Cavusoglu E, Chopra V, Gupta A, et al. Relation between red blood cell distribution width (RDW) and all-cause mortality at two years in an unselected population referred for coronary angiography. Int J Cardiol 141: 141-146, 2010.

18. Jackson CE, Dalzell JR, Bezlyak V, et al. Red cell distribution width has incremental prognostic value to B-type natriuretic peptide in acute heart failure. Eur J Heart Fail 11: 1152-1154, 2009.

19. Fukuta H, Ohte N, Mukai S, et al. Elevated plasma levels of Btype natriuretie poptide but not $\mathrm{C}$-reactive protein associated with higher red cell distribution width in patients with coronary artery disease. Int Heart J 50: 301-312, 2009.

20. Dabbah S, Hammerman H, Markiewicz W, Aronson D. Relation between red cell distribution width and clinical outcomes after acute myocardial infarction. Am J Cardiol 105: 312-317, 2010.

(C) 2011 The Japanese Society of Internal Medicine http://www.naika.or.jp/imindex.html 